\title{
Experience of cardioverter-defibrillators inserted without thoracotomy: evaluation of transvenously inserted intracardiac leads alone or with a subcutaneous axillary patch
}

Luc Jordaens, Jan-Willem Trouerbach, Patrick Vertongen, Luc Herregods, Jan Poelaert, Guido Van Nooten

\begin{abstract}
Objectives-To compare the efficacy of a purely transvenous cardioverter-defibrillator (ICD) system with that of a system with a supplementary subcutaneous patch. To evaluate clinical follow up of these lead arrangements that do not require thoracotomy.

Design-A simplified defibrillation protocol to test two different lead arrangements during implantation, with routine clinical follow up after implantation.

Setting-Tertiary referral centre for treatment of arrhythmia.

Patients-22 consecutive patients selected for implantation of an ICD because of life-threatening ventricular arrythmias (ventricular fibrillation or sustained ventricular tachycardia) of whom 20 entered the test protocol.

Intervention-Implantation of an ICD with transvenously inserted intracardiac leads and a subcutaneous patch and assessment of effective defibrillation followed by testing of the purely transvenous approach.
\end{abstract}

Main outcome measures-Reproducible conversion of ventricular fibrillation to sinus rhythm at a certain energy level, providing a safety margin of at least $10 \mathrm{~J}$ for both lead arrangements. Confirmation of efficacy during clinical follow up (mean 6 months).

Results-A transvenous lead system combined with a subcutaneous axillary patch was implanted in 20/22 patients and it provided adequate and acceptable energy levels. In 10/20 tested patients a purely transvenous lead configuration provided an acceptable safety margin as well. Nine patients had clinical recurrences: all these arrhythmias were successfully converted.

Conclusion-A transvenous lead system was sufficient in $50 \%$ of the patients at the time of implantation. Data on long-term clinical follow up of this arrangement are not available. The approach without thoracotomy with a subcutaneous patch is feasible and effective in most patients selected for ICD treatment.

(Br Heart f 1993;69:14-19)
The implantable cardioverter-defibrillator (ICD) had been a major improvement in the treatment of patients with life- threatening ventricular arrhythmias. Survival after a first episode of ventricular tachyarrhythmia was better than that in historical series, and sudden death after implantation was uncommon. $^{1-2}$ Implantation of an ICD with sternotomy or thoracotomy is associated with a mortality risk of about $5 \% \cdot \cdot^{3-4}$ This is a major limitation to clinical application. Patients with depressed left ventricular function and those who have had previous cardiac surgery or who undergo it during implantation seem to be especially susceptible to complications. Less invasive approaches have been developed in an attempt to reduce this procedure related mortality. ${ }^{5-7}$ Mirowski recognised in a very early stage of clinical development that not every ventricular arrhythmia could be converted to sinus rhythm with the available energy generated from the capacitors of the ICD. ${ }^{8}$ When transvenous, endocardiac leads or subcutaneous patches (with a smaller electrode surface or a greater distance from the heart) are used to deliver the defibrillating shocks, this problem will become more important than it is when epicardiac patches are used in a classic approach with sternotomy or thoracotomy. ${ }^{9} \quad$ Therefore, defibrillation threshold testing should be performed to ensure the presence of a safety margin between the energy level required to convert ventricular fibrillation and the maximal energy output of the device. This implies that the advantage of a less invasive implantation has to be balanced against the possibility that shocks will be less effective. After the first case report of an implantation without thoracotomy, the development of lead and patch systems was slow. ${ }^{10} \mathrm{We}$ describe our experience with two systems that do not need thoracotomy: the PCD 7217 with non-thoracotomy-leads of Medtronic and the Ventak 1600 and Ventak 1700 series of Cardiac Pacemakers Inc (CPI) that can be used with the Endotak lead system. ${ }^{112}$

Patients and methods

PATIENTS

Table 1 summarises the characteristics of the 22 patients (18 men and four women; mean age 60 (range 34-73)) referred for implanta- 
Table 1 Clinical data

\begin{tabular}{|c|c|c|c|c|c|c|c|c|c|}
\hline $\begin{array}{l}\text { Case } \\
\text { No }\end{array}$ & Sex & $\begin{array}{l}\text { Age } \\
(y r)\end{array}$ & $\begin{array}{l}\text { Cardiac } \\
\text { disease }\end{array}$ & Arrhythmias & $\begin{array}{l}\text { Previous } \\
\text { surgery }\end{array}$ & $\begin{array}{l}\text { Associated } \\
\text { arrhythmia }\end{array}$ & $\begin{array}{l}\text { Results } \\
\text { of PES }\end{array}$ & $\begin{array}{l}E F \\
(\%)\end{array}$ & $\begin{array}{l}\text { No of } \\
\text { previous } \\
\text { drug trials }\end{array}$ \\
\hline 1 & $\mathbf{M}$ & 72 & CAD & VT & An & - & SMVT & 28 & 3 \\
\hline 2 & $\mathrm{~F}$ & 55 & CAD & VF1 & - & - & $\mathrm{VFl}$ & 25 & 1 \\
\hline 3 & $\mathbf{M}$ & 57 & CAD & VT & C & - & SMVT & 28 & 3 \\
\hline 4 & $\mathbf{M}$ & 67 & CAD & $\mathrm{VT}$ & - & - & NI & 52 & 0 \\
\hline 5 & $F$ & 46 & CMP & VF & - & - & NI & 52 & 1 \\
\hline 6 & $\mathbf{F}$ & 52 & CAD & VF & C & - & VFl & 44 & 3 \\
\hline 7 & $\mathbf{M}$ & 72 & CAD & VT & - & - & SMVT & 41 & 2 \\
\hline 8 & $\mathbf{M}$ & 53 & - & VF & - & - & NI & 61 & 0 \\
\hline 9 & M & 34 & ARVD & VT & - & - & SMVT & 61 & 5 \\
\hline 10 & M & 54 & CAD & VF & C & AVB3 & VFl & 45 & 1 \\
\hline 11 & M & 51 & CAD & VT/VF & - & - & SMVT & 37 & 1 \\
\hline 12 & M & 65 & CMP & VT & - & - & $\mathrm{VFl}$ & 55 & 1 \\
\hline 13 & M & 70 & CAD & VT/VF & - & - & SMVT & 20 & 5 \\
\hline 14 & M & 61 & CAD & VT/VF & - & - & SMVT & 14 & 2 \\
\hline 15 & $\mathbf{M}$ & 73 & CAD & VT/VF & - & - & SMVT & 15 & 4 \\
\hline 16 & $\mathbf{M}$ & 68 & CAD & VF & - & & Not done & 53 & 0 \\
\hline 17 & $\mathrm{~F}$ & 66 & Ao & VT & AR & AVB3 & SMVT & 68 & 2 \\
\hline 18 & M & 58 & CAD & VT & - & - & SMVT & 38 & 3 \\
\hline 19 & M & 65 & CAD & VT & C & - & SMVT & 50 & 1 \\
\hline 20 & M & 55 & CAD & VT/VF & - & - & SMVT & 16 & 1 \\
\hline 21 & M & 67 & CAD & VT/VF & C & CAF & SMVT & 31 & 1 \\
\hline 22 & $\mathbf{M}$ & 68 & CAD & VT/VF & C & - & SMVT & 32 & 1 \\
\hline
\end{tabular}

An, aneurysm; Ao, aortic disease; AR, aortic replacement; ARVD, arrhythmogenic right ventricular dysplasia; AVB3, complete heart block; $C$, coronary artery bypass grafting; $C A D$, coronary artery disease; $C A F$, chronic atrial fibrillation; CMP, cardiomyopathy; EF, ejection fraction; NI, not inducible; PES, programmed electrical stimulation; SMVT, sustained monomorphic ventricular tachycardia; VF, ventricular fibrillation; $\mathrm{VFl}$, ventricular flutter.

tion up to the end of December 1991. The mean left ventricular ejection fraction determined with technetium-99 pertechnetate angiography was $38 \%$ (range $15 \%-68 \%$ ). Coronary artery disease was present in 17 patients, three had cardiomyopathy, one had valvar disease, and in the remaining patient no structural heart disease could be established with certainty. The presenting arrhythmia was ventricular tachycardia in nine patients and ventricular fibrillation with or without ventricular tachycardia in 12 . In one patient the arrhythmia was ventricular flutter. During programmed electrical stimulation a ventricular arrhythmia could be induced in 17 patients. Ten patients had been receiving antiarrhythmic drugs (including amiodarone in six) before the arrhythmic event leading to implantation. One patient with two configurations of ventricular tachycardia arising from the right ventricular outflow tract underwent ablation but had recurrences. Antiarrhythmic drug treatment was given after the electrophysiological study in 11 patients, and fast ventricular tachycardia remained inducible in all these patients. Treatment was continued in five patients to avoid frequent episodes of tachycardia, but in five other patients amiodarone treatment was discontinued before implantation. Two patients had a history of bradycardia: one had transient complete heart block. The other underwent ablation of the atrioventricular node before implantation of the ICD because of atrial fibrillation associated with fast conduction of the ventricular tachycardia. The ethics committee approved of the implantation of these investigational systems and all patients gave informed consent.

\section{DEVICES}

Two different types of cardioverter-defibrillators were used. The PCD 7217 developed by Medtronic provides the usual cardioverterdefibrillator capabilities, an additional pacing facility for bradycardia support, and burst and ramp pacing algorithms for tachycardia termination. ${ }^{11}$ The PCD recognises two tachyarrhythmia zones. Rate stability is an additional programmable variable. The device stores the number of episodes of tachycardia or fibrillation and counts the shocks or antitachycardial pacings that are given. It also memorises the RR intervals before and after the last treatment. The shock energies can be programmed from 0.2 to $34 \mathrm{~J}$. Bidirectional shocks can be given sequentially or simultaneously. The Ventak 1600 and Ventak 1700 devices were developed by CPI for use in combination with the Endotak lead system. ${ }^{12}$ The Ventak 1700 (PRx) has three rate zones. Additional sensing variables are "turning point morphology" and rate stability. Antitachycardia pacing is possible in the two lower rate zones, and shocks are possible in all three zones. The device has a shock range of 0.1-34 J, and also has bradycardia pacing. It stores information on episodes, detection criteria that were met, and $R R$ intervals immediately before and after treatment.

\section{CATHETERS AND PATCHES}

The Medtronic system consists of a tripolar transvenous cardioverter-defibrillator lead, with a screw in bipolar sensing and pacing tip (Model 6884 or 6966). The distal coil has a $290 \mathrm{~mm}^{2}$ platinum alloy surface, and is placed in the right ventricular apex. Also a second lead (model 6881 or 6963 ) is provided. This can be advanced to the superior vena cava or inserted in the coronary sinus. An oval patch (model 6895 or 6999), with a smooth silicone rubber coating, covering a $660 \mathrm{~mm}^{2}$ coil is used for subcutaneous implantation.

The Endotak system of CPI has a single tripolar catheter with a tined pacing tip. There is defibrillation coil near the tip and a second coil $10-16 \mathrm{~cm}$ from the tip. The second coil is placed in the superior vena cava. A 


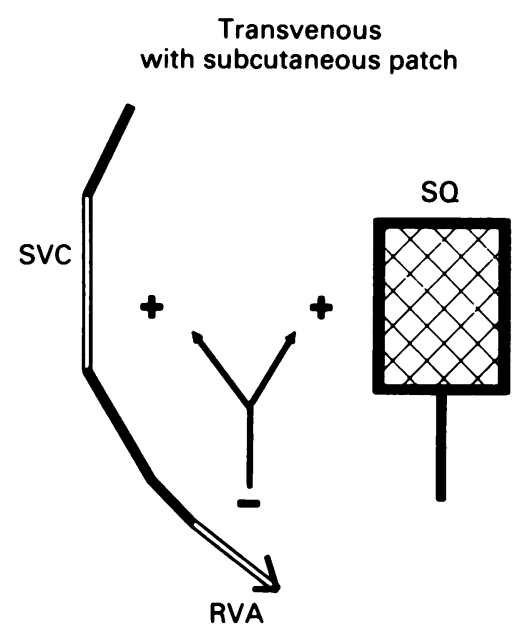

Transvenous

with subcutaneous patch

RVA
Transvenous lead alone

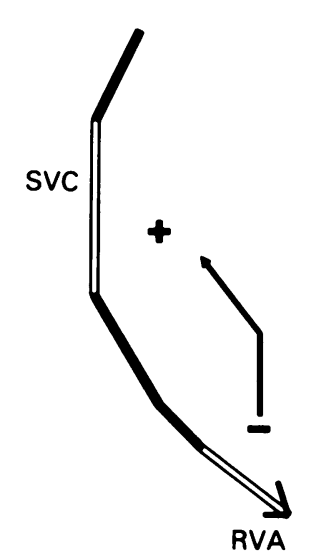

Figure 1 Drawing of a lead and patch triple electode system (resembling the Endotak system), indicating the direction of shock in both arrangements tested: lead and subcutaneous (SQ) patch and transvenous lead alone. RVA, right ventricular apex: SVC, superior vena cava. rectangular patch is provided for subcutaneous use. A Y-shaped adapter connects this system to the ICD.

\section{IMPLANTATION}

In all patients depending on the selected system, one or two transvenous leads were placed in the heart after dissection of the cephalic vein or puncture of the subclavian vein. After the lead was positioned in the right ventricular apex the pacing threshold was determined on the tip electrodes. If the pacing threshold was too high, or when the sensed signal was too small, the lead was repositioned. Where the Medtronic system was used, the second lead was always positioned in the superior vena cava. After correct placement of the transvenous catheter system, an anteroposterior incision was made in the left subaxillary region for placement of the patch. Then defibrillation was tested. If the energy levels were acceptable the lead system was connected with the ICD that was positioned in a pocket in the abdominal wall. The device was then activated during the operation to ensure proper connections, correct sensing, and effective shock delivery. The implantation procedure in the first two patients was different form the general protocol: firstly, testing was started in these two patients with the transvenous system alone and secondly a large external patch on the skin in the subaxillary position was used for testing instead of the subcutaneous patch.

\section{DEFIBRILLATION TESTING}

After the first two patients, we adhered to our own modified protocol. Monophasic shocks were used. After placement of the transvenous lead system and subcutaneous patch, ventricular fibrillation was induced with alternating current. After at least 10 seconds of ventricular fibrillation, a $20 \mathrm{~J}$ shock (Endotak systems) or an $18 \mathrm{~J}$ shock (PCD systems) was given by an external cardioverting-defibrillating device. In stage I we tested an arrange- ment in which the right ventricular coil acted as the cathode and both the coil in the superior vena cava and the patch acted as the anode. If simultaneous bi-directional monophasic shocks (fig 1) did not produce cardioversion we gave a maximal back up shock $(40 \mathrm{~J})$ through the implanted system. If this failed again, a $400 \mathrm{~J}$ shock was given through external defibrillation patches. If the first shock succeeded, two more episodes had to be converted with similar shock energy levels to meet standard implantation criteria. After failure of one shock, an alternative patch position was selected to achieve at least three out of four successful defibrillations with 18 or $20 \mathrm{~J}$ in this new arrangement. If this was not feasible, we accepted three successful defibrillations with 24 or $25 \mathrm{~J}$. Thereafter, in stage II we assessed the feasibility of transvenous shocks between the cathode in the right ventricular apex and the anode in the superior vena cava alone (fig 1). To limit the duration of testing, we stopped after two successful successive defibrillations. Testing was only performed at energy levels up to 24 or $25 \mathrm{~J}$. Where a purely transvenous system would have been acceptable, however, the subcutaneous patch was always left in place, with programming for bi-directional shocks.

\section{Results}

INITIAL EXPERIENCE

In the first two patients no acceptable defibrillation threshold was found with a purely transvenous system, nor with any other arrangement without thoracotomy, including those with a lead in the coronary sinus. The first patient had a history of aneurysmectomy. The threshold for reproducible defibrillation with two large epicardiac patches was less than $18 \mathrm{~J}$. The second patient also could not be defibrillated with either of the non- thoracotomy arrangements; after two large epicardiac patches were placed, defibrillation was possible with $18 \mathrm{~J}$.

\section{BI-DIRECTIONAL DEFIBRILLATION}

(SUBCUTANEOUS PATCH AND TRANSVENOUS LEAD SYSTEM)

The last 20 patients who were tested with the two-stage protocol could be managed with the combination of a subcutaneous (subaxillary) patch and a transvenous lead system. Eleven patients were defibrillated three times with 18 or $20 \mathrm{~J}$. In two patients, three out of four episodes, and in one, two out of three episodes were defibrillated at this energy level. This last patient (No 14) had a pneumothorax and it was decided to stop further testing. After one week he was defibrillated three times with $18 \mathrm{~J}$ during testing before discharge. Another patient (case 12) was treated with $d$-sotalol. All attempts to convert ventricular fibrillation resulted in a very slow non-sustained ventricular tachycardia without haemodynamic repercussions. It always ended spontaneously within 70 seconds. He underwent testing when $d$-sotalol had washed 


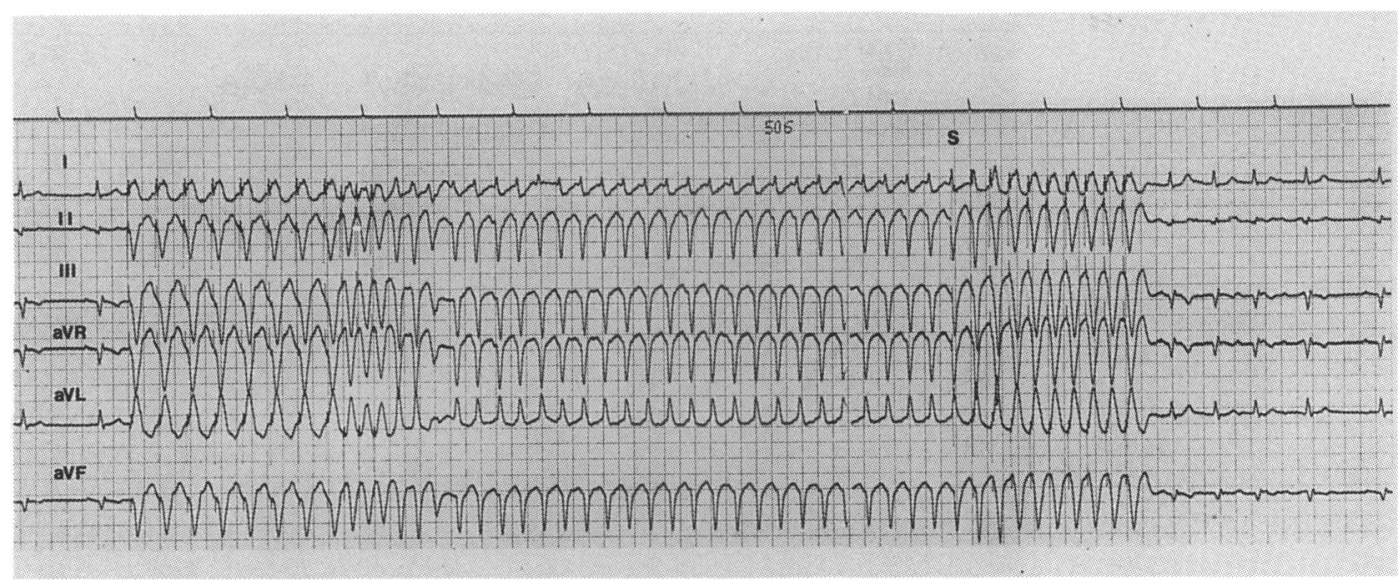

Figure 2 Ending tachycardia with pacing. Induction of sustained tachycardia (by the software of the implanted PCD). After seven seconds the arrhythmia is converted to sinus rhythm with a burst of 10 stimuli (S).

out and sinus rhythm was restored without any intervening ventricular arrhythmia. In the other five patients testing was stopped when the implantation criteria were met with a safety margin of $10 \mathrm{~J}$.

\section{INTRACARDIAC DEFIBRILLATION}

\section{(TRANSVENOUS LEAD ALONE)}

The two stage testing was completed in this sequence in $18 / 20$ patients (excluding the first two patients in whom the non- thoracotomy arrangements were unsuccessful). The protocol was not applied in the patient with the pneumothorax and the patient given $d$-sotalol. In seven patients sinus rhythm was restored by a shock of 18 or $20 \mathrm{~J}$ in the two defibrillation trials. In three additional patients, defibrillation was reproducible with 24 or $25 \mathrm{~J}$. Previous cardiac surgery, left ventricular ejection fraction, and previous treatment with class III antiarrhythmic drugs did not seem to influence the effects in this small series.

\section{COMPLICATIONS}

The first patient from the series, who underwent thoracotomy, died of multiple organ failure six days after the intervention. No others died perioperatively. One had a left apical pneumothorax after puncture of the subclavian vein. It resolved spontaneously. In one patient with a history of severe ischaemic heart disease myocardial infarction recurred six days after implantation of the device.

\section{PROGRAMMING AND TESTING BEFORE}

DISCHARGE

For all patients energy levels were programmed to be $34 \mathrm{~J}$ from the first shock onwards. No low energy shocks were programmed. Bradycardia pacing was programmed for 30 to 50 beats a minute. Anti-tachycardia pacing was adapted to the findings from the studies before implantation in patients with inducible tachycardia. Nineteen out of 21 patients (including the surviving patient with thoracotomy) had a non-invasive test before discharge. In 18 patients, ventricular fibrillation was induced. All were defibrillated from this arrhythmia with the first shock ( $34 \mathrm{~J}$ ) except patient 2 who needed implantation via a thoracotomy. In this patient two shocks were needed. In three patients the study was focused on induction of the clinically important ventricular tachycardia and on the programming of

Table 2 Implantation data and follow up

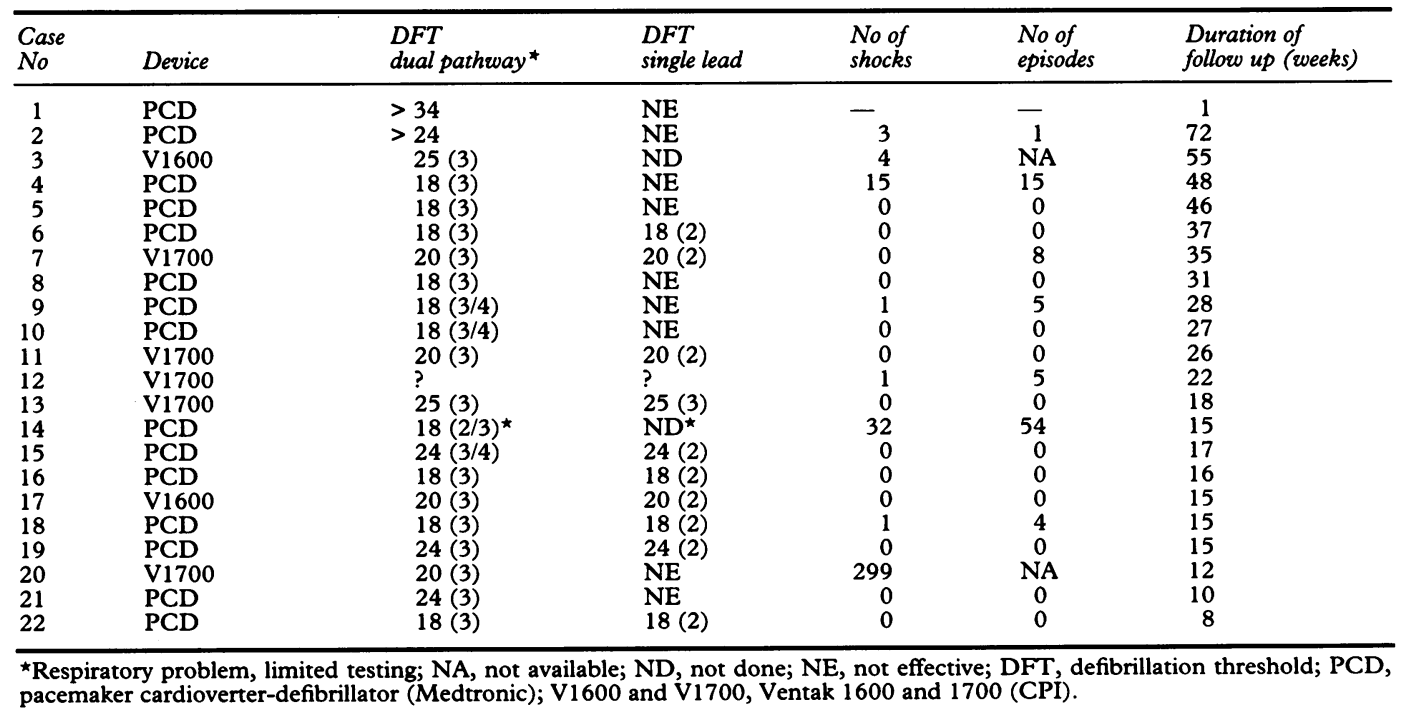




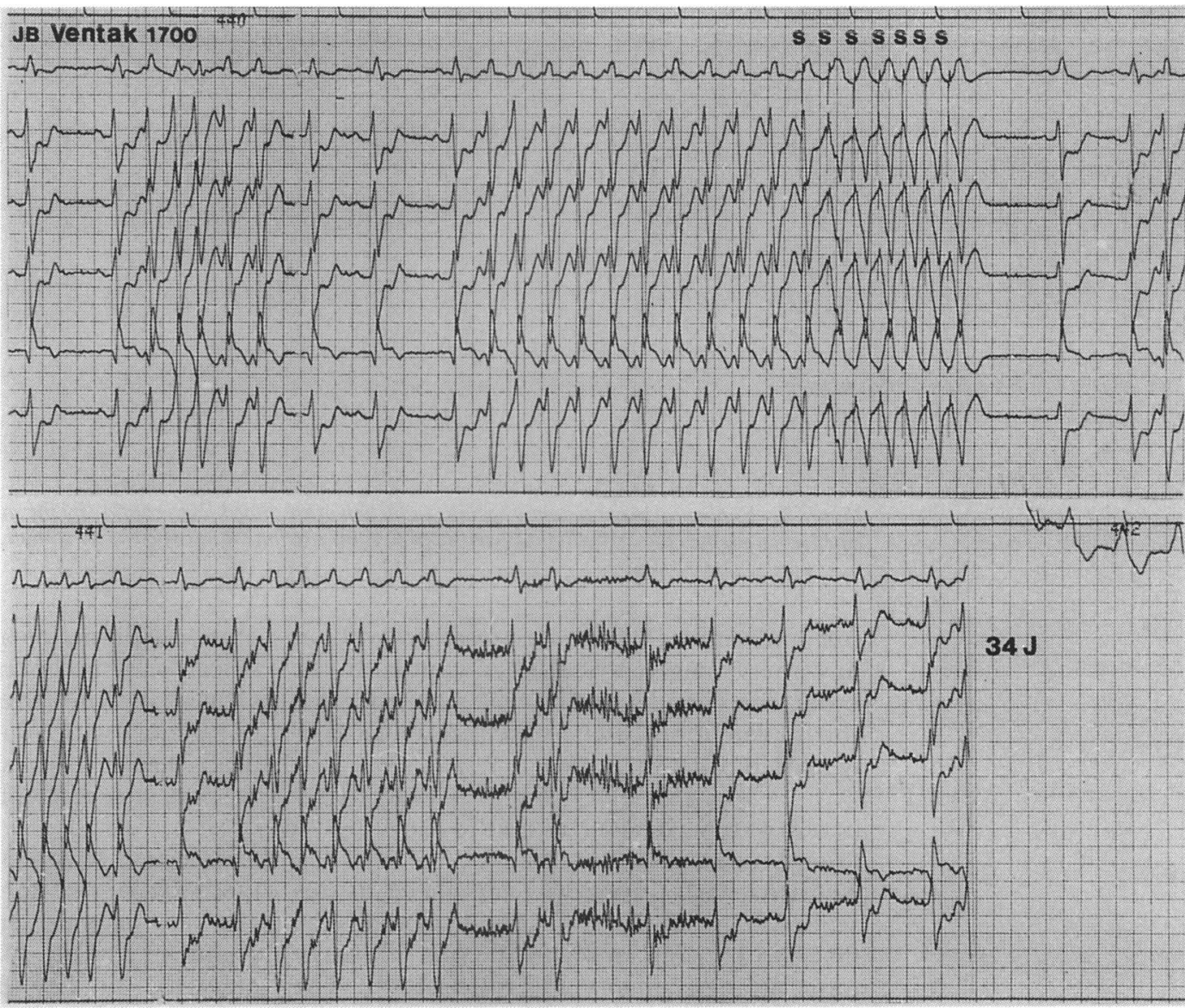

Figure 3 Recurrent ventricular tachycardia was terminated by pacing but started again after two seconds. $A$ shock of 34 $\mathcal{F}$ was delivered after spontaneous termination of tachycardia. This occurred despite programming of the device in "noncommitted" mode. It is not clear whether there was a software problem on this occasion.

the anti-tachycardia pacing modes (fig 2). In two patients antiarrhythmic the drug treatment was changed and non-invasive testing was repeated. In one patient (case 20) testing before discharge was repeated because of the clinical occurrence of frequent shocks.

\section{CLINICAL FOLLOW UP}

Follow up was completed on 29 February 1992. It ended with the last scheduled bimonthly follow up visit or with death (case 1) or heart transplantation (case 14). Mean follow up duration was 26 (range 1-72) weeks. Table 2 shows the number of spontaneous episodes of tachyarrhythmia and the number of shocks. Nine patients experienced episodes during follow up. Eight of these patients received shocks. Six had episodes that were ended by the programmed pacing algorithms. Only two patients required more than one shock for an episode. One of them (patient 20), had several episodes with multiple shocks and received drug treatment ( $80 \mathrm{mg}$ sotalol three times daily) to suppress recurrences after defibrillation. This did not prevent premature exhaustion of the ICD (fig 3 ). It was replaced after ablation of the tachycardia. Patient 4 with a history of multiple myocardial infarctions had paroxysmal complete heart block during the routine Holter monitoring after implantation, with correct pacemaker function. He had another infarc- tion five months after implantation, and received multiple shocks during this event. Patient 14 had several episodes of ventricular tachycardia ended both by pacing and shocks. He underwent successful heart transplantation after recurrent pulmonary oedema.

\section{Discussion}

Our early experience with the non-thoracotomy approach was disappointing. The initial selection of two difficult cases certainly played a part in the fact that we had to proceed to thoracotomy. Further experience showed that the non-thoracotomy approach was feasible in most patients with techniques currently available. This accords with most other available data. ${ }^{12-14}$ Moreover, we could identify a large subset of patients who could potentially be treated with an intracardiac lead arrangement without a subcutaneous patch. It is known that such patches are responsible for much of the morbidity recorded in recently compiled databases (erosion, seroma, haematoma), and therefore the transvenous leads only approach becomes even more attractive. ${ }^{15} \mathrm{We}$ thought that it was not safe to have a lead system without a patch in these patients. We had already set a safety margin of $10 \mathrm{~J}$ because cardiac disease continues after implantation. During the follow up, two of our patients had a myocardial 
infarction, and another had serious functional deterioration leading to transplantation. Progression of cardiac disease could substantially change the amount of energy required to defibrillate a heart. Also intracardiac leads can be dislodged.

Defibrillation threshold testing remains necessary as it can identify patients at high risk of subsequent problems. ${ }^{16}$ Testing is not, however, without risks. it has been suggested that defibrillation threshold testing should be restricted as much as possible, to prevent cardiac and cerebral problems. ${ }^{17-18}$ If many defibrillations are given early in the testing, the condition of the patient can be affected, and subsequent possible effective arrangements of a lead and subcutaneous patch may fail. This was a possible explanation for the failure in our first two patients. It was therefore decided to start testing with the lead and subcutaneous patch arrangement, which is probably the safest and most effective in a first stage. ${ }^{15}$ The feasibility of the lead alone arrangement was assessed in the second stage. The morbidity with the revised testing and implantation protocol was negligible. Many drawbacks of early devices are now avoided by the new systems we used. ${ }^{19}$ Bradycardia and antitachycardia pacing functions are incorporated. This allows ending of some tachycardias without shocks and without pain. Furthermore, the life span of the device is extended and sensing is improved by some features, including rate regularity. The implantation is less invasive, and we assume that the cost will decrease.

The final evaluation of the benefit of ICD treatment depends on the follow up: clinical recurrences of arrhythmias have to be reliably converted to sinus rhythm. ${ }^{20}$ About $41 \%$ of our patients received treatment with shocks or pacing in a clinical setting. None of these patients died suddenly over a short follow up period of 26 weeks. This supports the hypothesis that ICDs that do not require thoracotomy are indeed effective. We hope that this will allow us to treat patients in whom thoracotomy and epicardiac patch placement would be poorly tolerated.

1 Mirowski M, Reid PR, Winkle RA, Mower MM, Watkin L Jr, Stinson EB, et al Mortality in patients with $\mathrm{L}$ Jr, Stinson EB, et al. Mortality in patients with
implanted defibrillators. Ann Int Med 1983;98:585-8.
2 Winkle RA, Hardwin Mead R, Ruder MA Gaudiani VA, Smith NA, Buch WS, et al. Long-term outcom with the automatic implantable cardioverter-defibrillator. $\mathcal{F}$ Am Coll Cardiol 1989;13:1353-61.

3 Guarnieri T, Levine JH, Griffith LS, Veltri EP. When "sudden cardiac death" is not so sudden: lessons learned from the automatic implantable defibrillator. Am Heart J 1988;115:205-7.

4 Kim SG, Fisher JD, Furman S, Gross J, Zilo P, Roth JA et al. Benefits of implantable defibrillators are overestimated by sudden death rates and better represented by the total arrhythmic death rate. $\mathcal{f}$ Am Coll Cardiol 1991; 17:1587-92.

5 Mirowski M, Mower MM. Transvenous catheter defibrillation for prevention of sudden cardiac death. $\mathcal{F} \mathrm{Am} \mathrm{Coll}$ Cardiol 1988;11:371-2

6 Winkle RA, Bach SM, Hardwin Mead R, Gaudiani VA, Stinson B, Fain ES, et al. Comparison of defibrillation efficacy in humans using a new catheter and superior vena cava spring-left ventricular patch electrodes. $\mathfrak{F} \mathrm{Am}$ vena cava spring-left ventricular

7 Zipes DP, Heger JJ, Miles WM, Mahomed Y, Brown JW, Spielman SR, et al. Early experience with an implantable cardioverter. $N$ Engl f Med 1984;311: 485-90.

8 Winkle RA, Stinson EB, Bach SM Jr, Echt DS, Oyer P, Armstrong K. Measurement of cardioversion/defibrillation thresholds in man by a truncated exponential waveform and an apical patch-superior vena caval spring electrode configuration. Circulation 1984;69:766-71.

9 Troup PJ, Chapman PD, Olinger GN, Kleinman LH The Implanted defibrillator: relation of defibrillating lead configuration and clinical variables to defibrillation threshold. $₹$ Am Coll Cardiol 1985;6:1315-21.

10 Saksena S, Parsonnet V. Implantation of a cardioverter/ defibrillator without thoracotomy using a triple electrode system. $\mathcal{F A M A}$ 1988;259:69-72.

11 Fromer M, Schläpfer J, Fischer A, Kappenberger L Experience with a new implantable pacer-, cardioverterdefibrillator for the therapy of recurrent sustained ventricular tachyarrhythmias: a step toward a universal ventricular tachyarrhythmia control device. $P A C E$ 1991;14:1288-98.

12 Paul VE, Anderson M, Jones S, Skehan DJ, Camm AJ Ward DE. Cardiologist implanted cardioverter/defibrillators: early experience of three systems. European Fournal of Cardiac Pacing and Electrophysiology 1991; 1:27-38.

13 Singer I, Austin E, Nash W, Gilbo J, Kupersmith J. The initial clinical experience with an implantable carinitial clinical experience with an implantable car1991;14:1119-28.

14 Saksena S, Mehta D, Krol RB, Tullo MG, Saxena A Kaushik R, et al. Experience with a third-generation implantable cardioverter-defibrillator. $\mathrm{Am} \mathcal{\exists}$ Cardio 1991;67:1375-84.

15 Lindemans FW, van Binsbergen E, Connolly D. Clinical evaluation report. European PCD ${ }^{\mathrm{TM}}$ study. Patients with Transvene ${ }^{\mathrm{TM}}$ lead systems. Maastricht: Bakken Research Center, 1991;32-3.

16 Pinski SL, Vanerio G, Castle LW, Morant VA, Simmons TW, Trohman RG, et al. Patients with a high defibrillation threshold: clinical characteristics, management, and tion threshold: clinical characteristics,

17 Block $M$, Borggrefe $M$, Hammel $D$, Isbruch $F$, Scheld HH, Breitharddt G. Intra-operative testing for PCD implantation. In: Lindemans FW, Rankin IR, Vegter J, eds. Proceedings of the Symposium on PCD Clinical Results. Maastricht: Bakken Research Center, 1991;17-21.

18 Singer I, Van Der Laken J, Edmonds HL, Slates AD, Austin E, Shields CB, et al. Is defibrillation testing safe? PACE 1991;14:1899-1904.

19 Holt PM, Crick JCP, Sowton E. Experience with an automatic implantable cardioverter defibrillator. Lancet 1987;i:551-3.

20 Jordaens L, Waleffe A, Derom F, Rodriguez LM, Clement DL, Kulbertus $H$. First experience with the implantable cardioverter-defibrillator: 90 patientimplantable cardioverter-defibrillator: 90 patient-
months of follow-up. Acta Clin Belg 1988;43:209-18. 